\title{
Téoros
}

Revue de recherche en tourisme

\section{L’insécurité urbaine}

\section{Un mal qui paralyse les femmes}

\section{Renée Rouleau}

Volume 16, numéro 3, automne 1997

Femmes et tourisme

URI : https://id.erudit.org/iderudit/1073316ar

DOI : https://doi.org/10.7202/1073316ar

Aller au sommaire du numéro

Éditeur(s)

Université du Québec à Montréal

ISSN

0712-8657 (imprimé)

1923-2705 (numérique)

Découvrir la revue

Citer cet article

Rouleau, R. (1997). L’insécurité urbaine : un mal qui paralyse les femmes.

Téoros, 16(3), 25-28. https://doi.org/10.7202/1073316ar

Ce document est protégé par la loi sur le droit d'auteur. L'utilisation des services d'Érudit (y compris la reproduction) est assujettie à sa politique d'utilisation que vous pouvez consulter en ligne.

https://apropos.erudit.org/fr/usagers/politique-dutilisation/
Cet article est diffusé et préservé par Érudit.

Érudit est un consortium interuniversitaire sans but lucratif composé de l'Université de Montréal, l'Université Laval et l'Université du Québec à Montréal. Il a pour mission la promotion et la valorisation de la recherche. https://www.erudit.org/fr/ 


\section{L'INSÉCURITÉ URBAINE}

\section{UN MAL QUI PARALYSE LES FEMMES}

Renée Rouleau, consultante

L'objet de cet article consiste à susciter l'intérêt des intervenants de l'industrie touristique au phénomène de l'insé́curité que suscitent les espaces urbains, les lieux publics ou les destinations voyage.

Au départ, il est essentiel de distinguer lá violence de l'insécurité. La première consiste en des actes tandis que la seconde, liée aux conséquences ${ }^{1}$ de ces actes, se qualifie d'émotion. Cette émotion, trop souvent laissée pour compte, joue pourtant un rôle important dans les décisions des consommateurs de fréquenter un lieu, de se promener dans un quartier ou de voyager dans un pays plutôt que dans un autre.

Au Québec, le fait de s'intéresser plus spécifiquement au phénomène de l'insécurité dans la prévention de la violence provient de l'implication des groupes de femmes canadiennes. Ces féministes ont prôné le droit des femmes à l'accès aux lieux publics et toute sécurité dans leur quartier le soir tout comme pour la plupart des hommes. Elles ont identifié les éléments de l'environnement qui leur font gếnéralement peur. Elles ont sensibilisé les organismes publics et les corporations à leur réalité.

Mais, l'insécurité....

De quoi se nourrit-elle?

Abordons le sujet par un exemple en utilisant Montréal, une ville perçue à la fois comme dangereuse par certains et sécuritaire par d'autres. On entend souvent dire que les touristes qui fréquentent Montréal, la choisissent parce qu'ils s'y sentent en sécurité, bien que d'autres villes puissent avoir beaucoup d'attraits. On la trouve propre, non violente et les gens sont sympathiques. Les études tendent à démontrer que Montréal est une des villes les plus sécuritaires au monde. Dans les faits, si vous demandez à monsieur-toutle-monde, à un Québécois qui n'habite pas Montréal, s'il se considère en sécurité lorsqu'il y vient, il vous répondra que c'est dangereux, qu'il y a beaucoup trop de violence pour se sentir en sécurité. Même si des études proclament des villes «sécuritaires», le sentiment d'insécurité lui est une réalité, une émotion personnelle qu'il faut considérer. Qui a raison? Dans le domaine de la sécurité urbaine, il faut considérer, au-delà des statistiques, les espaces et lieux publics.

Le sentiment de sécurité ou d'insécurité est une réalité qu'il faut comprendre. Ce dernier a énormément d'influence sur l'économie locale, sur la viabilité des quartiers centraux, sur le développement des banlieues et sur le tourisme.

Poursuivons notre exemple, et demandons-nous pourquoi ce sentiment d'insécurité persiste chez de nombreux Québécois à l'égard de Montréal. Ce sentiment serait-il nourri par ce que l'on voit et entend des médias? Serait-ce que l'on compare Montréal à notre propre milieu perceptions humaines par rapport aux de vie (en banlieue ou à la campagne)? En fait, plusieurs raisons sont en cause tant sur le plan individuel que sur le plan collectif.

La perception d'être vulnérable et l'exposition aux actes de violence sont d'importants facteurs qui influent sur le plan individuel le sentiment d'insécurité. Sur le plan collectif, l'insécurité se nourrit des manifestations de violence dans les médias et le cinéma ainsi que dans la façon dont on l'aborde. Aussi, l'aménagement urbain, la propreté, la fréquentation des lieux publics, l'appropriation de l'espace semiprivé et public par les résidents et les propriétaires, la prise en charge locale (empowerment) sont tous des aspects importants qui influencent le sentiment de sécurité sur le plan collectif.

Abordons plus en détails ces facteurs d'insécurité.

\section{FACTEURS INDIVIDUELS DE L'INSÉCURITÉ}

L'insécurité urbaine, cette peur étrange de sortir le soir, cette peur de quitter son chezsoi, cette peur qui paralyse le corps et l'esprit, apparaît plus importante chez certains groupes de la population.

Les premiers touchés par le sentiment paralysant de l'insécurité sont ceux qui ont déjà subi des actes de violence. Il y a aussi ces gens qui ont peur parce qu'ils connaissent une personne ou ont entendu parlé d'une personne qui a été victime de violence urbaine. Le sentiment d'insécurité est une plaie difficile à guérir. 
Généralement, une des caractéristiques psychologiques les plus importantes chez ceux qui ont peur c'est la perception d'être vulnérable, ce qui est le lot des personnes âgées, des personnes handicapées physiquement et de beaucoup de femmes. Selon l'Enquête sur la violence envers les femmes $^{2}, 60 \%$ des femmes sont inquiètes de marcher seule dans leur propre quartier lorsqu'il fait noir. Il ne faut pas croire que les hommes sont complètement immunisés: ils ont peur pour leurs enfants, leur conjointe, leurs parents âgés et aussi pour... eux-mêmes.

\section{FACTEURS COLLECTIFS DE L'INSÉCURITÉ}

\section{A) Le degré d'exposition collectif à des manifestations de violence: les médias}

L'insécurité urbaine n'est pas causée seulement par le fait d'avoir été victime ou de se sentir vulnérable. Les médias. rapportant les événements violents, contribuent aussi au sentiment d'insécurite dans la population. Ils prétendent pourtant représenter les intérêt du public à être informé. Certains, par ailleurs, utilisent des moyens médiatiques (photos vidéo) rendant les faits rapportés des épisodes spectaculaires. Ces moyens sont souvent exagérés par rapport aux valeurs que l'on devrait accorder à ces événements plutôt amoraux. Ce n'est pas que ces faits soient non-importants, au contraire, la violence doit être décriếe et non mis en spectacle. On parle plutôt (en langage déguisé) de marketing et de libre marché. Bien sûr, les gens aiment acheter le spectaculaire, ce qui sort de l'ordinaire. Les médias se défendent en disant que les gens aiment la violence et elle doit être présentée en spectacle. Ainsi, on vend non plus les faits mais une fabulation. Il faudrait, une fois pour toute, que les médias comprennent leurs rôles et responsabilités morales. Whe medium is the messagew, les fervents des médias se reconnaîtront. il faudrait que certains se posent la question aQuel est le message transmis dans la façon de présenter les faits violents? $\%$. S'il y a plus de violence dans les médias, les gens se sentiront moins en sécurité et seront portés à croire que dehors c'est dangereux, ils resteront donc chez eux et écouteront la télé. Dans les faits, la violence sert bien les médias.
Par ailleurs, si les médias rapportent des actes de violence par rapport à un lieu géographique, il est fort probable que les gens décident de ne pas fréquenter ces endroits. Pour la protection des touristes, cela peut s'avérer une bonne chose, mais, seulement si l'information diffusée n'est pas brouillée par l'exagération.

On peut donc dire que le tourisme peut être influencé par la façon dont les médias traitent les actes de violence.

\section{B) La connaissance d'un milieu ou l'idée que l'on s'en fait}

Les gens qui ne font que passer dans un quartier lisent les éléments extérieurs de l'environnement et se font une idée de cet environnement par rapport à ce qu'ils connaissent déjà (ou par ce qu'ils ont appris des médias). Les études ont démontré qu'entre deux quartiers à niveau de violence égal, les gens se sentent plus en sécurité dans le quartier où ils habitent parce qu'ils le connaissent.

La connaissance et la familiarité d'un milieu ${ }^{3}$ jouent pour beaucoup sur le sentiment de sécurité. Par ailleurs, pouvonsnous ainsi dire qu'en tourisme, plus les gens auront de l'information descriptive sur un environnement qu'ils s'apprêtent à visiter, plus ils se sentiront en sécurité lors de leur visite. Le sentiment de sécurité est aussi lié à la capacité d'un individu à se repérer facilement. L'expérience auprès des femmes a démontré que si elles savent où elles vont, leur sentiment de sécurité est accru.

\section{C) L'achalandage des lieux publics et la prise en charge locale}

Un environnement qui inspire la sécurité se caractérise par un bon achalandage. Et vice et versa, un bon achalandage inspire la sécurité. Le parc Lafontaine, par exemple, a été pointé par les femmes comme étant un endroit insécurisant le soir et elles l'évitent aux heures de noirceur. Toutefois, on peut constater que lorsqu"il y a des concerts, l'achalandage est important partout dans le parc et il se dégage un sentiment de sécurité.

Il arrive que certains lieux publics perdent le niveau d'achalandage critique et soient utilisés en exclusivité par des gangs de rue. Ces lieux publics perdent alors leur attrait et deviennent insécurisants. Petit à petit,

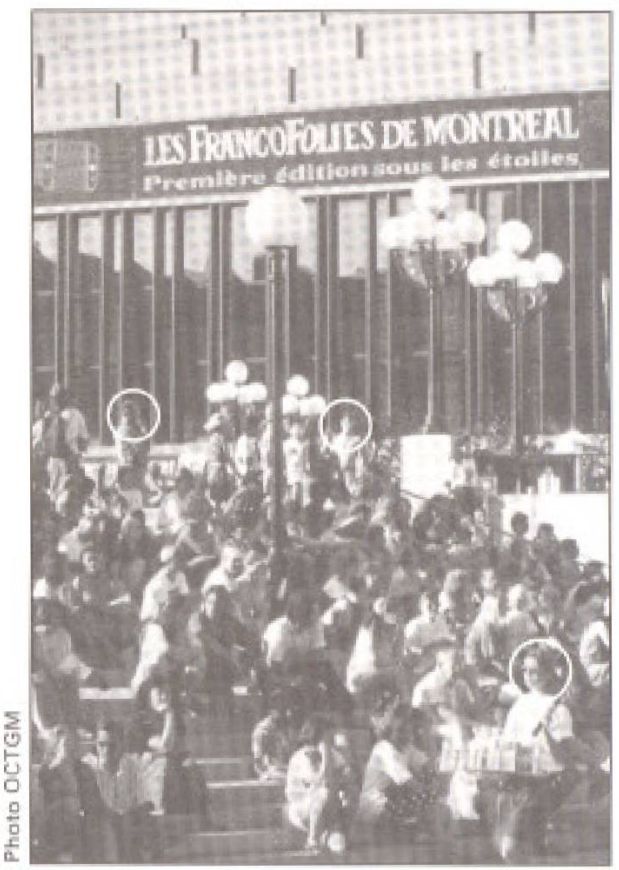

ils seront moins fréquentés. L'appropriation de l"espace par les citoyens ou par les responsables d'organisations d'événements est cruciale dans les cas où le risque d'abandon d'un site est grand. L'aménagement urbain peut jouer un rôle intéressant pour rendre les espaces publics propices à ce type d'appropriation, de prise en charge.

\section{D) L'aménagement, la propreté et les eléments de l'environnement}

Les études ont démontré que l'aménagement urbain et le type de fréquentation des espaces publics et privés sont liés à la peur d'être victime de violence. La liste des éléments urbains engendrant l'insécurité est longue: les terrains vacants; la présence de graffitis; les terrains en friches; les bâtiments inoccupés; les gangs de rue; la présence d'itinérants ou de mandiants' les stationnements mal éclairés; le manque d'indications dans les stationnements pour retrouver sa voiture; l'illisibilité des lieux (ne pas pouvoir se repérer facilement); les longs couloirs vides; les tunnels; les rues oû̀ les automobilistes ne peuvent s'inmobiliser; les déchets étalés; les zones industrielles; la présence policière; la présence de prostituées; etc.

Les groupes de femmes ont fait valoir l'importance d'etre en mesure: 1) de voir les éléments de l'environnement; 2) d'être vu par une présence humaine; 3) d'entendre; 4) d'être entendu; 5) de pouvoir avoir de l'aide rapidement; 6) de pouvoir fuir 


\section{DES ACTIONS}

\section{A TORONTO}

En 1984, le Metro Action Committee on Public Violence Against Women and Children (METRAC) a été créé, Celui-ci, principalement composé d'organismes communautaires, est financé par le Toronto Métropolitain. Il s'est affairé, en collaboration avec le Metro Police et le Toronto Transit Commission, à piloter des vérifications de la sécurité dans les transports publics et à développer des projets de sécurité sur les campus universitaires. Diverses publications ont été le fruit de cette collaboration dont *Moving Forward: Making Transit Safer for Women s et «Planning for Sexual Assault Prevention: Women's Safety in High Parks.

METRAC développa un guide «Women's Safety Audit Guiden qui a été l'inspiration de plusieurs projets au Canada. Ce guide, créé à 'tattention des groupes de femmes, est un outil de conscientisation et d'organisation communautaire qui cherche à les sensibiliser sur la fragilite de leurs peurs des endroits publics. Le guide propose une série de ren commandations à ce sujet. On y suggère une démarche de visite en groupe des lieux publics la nuit et de vérifier les éléments d'insécurité. À Toronto, le guide a été utilisé pour les parcs publics, les centres de loisirs, le transport en commun, les écoles, les projets résidentiels et les campus universitaires. Toronto est devenue la ville canadienne modèle en ce qui a trait aux projets de sécurité des femmes en milieu urbain. Elle a inspiré plusieurs projets de sécurité urbaine autant à Montréal, Ottawa, Vancouver qu’à Edmonton.

\section{A MONTRÉAL}

En 1989. la Ville de Montréal met sur pied le Comité inter-services Femmes et Ville qui réunit les membres de l'administration municipale. Mandaté par le Comité exécutif, il relève du Service des loisirs et du développement communautaire. Les travaux de ce comité ont portế sur l'accessibilité, les ser-

aisément. Pour les féministes, ces principes devraient guider les plans d'aménagement favorisant ainsi la sécurité des femmes.

\section{LES APPROCHES POUR PRÉVENIR LA VIOLENCE ET LA PEUR}

Malgré les liens qui existent entre eux, les phénomènes de la peur et de la violence vices de garde, le logement familial, le loisir et la sécurité des femmes à Montréal. En $s^{\prime}$ inspirant des travaux de METRAC, la Ville de Montréal a produit trois guides soit le * Guide d'enquête», le «Guide d'aménagement sécuritaire des stationnements» et le wGuide d'aménagement sécuritaire des ensembles résidentiels», Ceux-ci ont été distribues gratuitement aux organismes qui en faisaient la demande et ce, à l'echelle du Québec,

A l'automne 1992, a la suite de la première conférence montréalaise sur les femmes et la sécurité urbaine oJ'accuse la peurv, la Ville de Montréal avec la collaboration de groupes de femmes et de représentants d'organismes publics, met sur pied le Comité d'action femmes et sécurité urbaine (CAFSU). Le CAFSU, initiateur de plusieurs projets de sécurité urbaine compte parmi ses membres des représentants des divers groupes de femmes ainsi que ceux d'organismes publics tels le Service de la police, Tandem Montréal, le Service de transports en commun, la Régic régionale de la santé et des services sociaux. la Ville de Montréal, la Communauté urbaine de Montréal.

\section{AILLEURS AU QUÉBEC}

Une démarche d'interventions municipales à l'échelle provinciale a emboitté le pas en 1993 dans douze municipalités du Québec grace au programme «Aux portes des cités süresw financé par le Programme de promotion de la femme du Secrétariat d'État du Canada et mis en oeuvre par le Développement québécois de la sécurité des fémmes en milieu urbain. Les organisatrices du projet ont demandé à des groupes de femmes de douze municipalités du Québec d'établir des partenariats avec les représentants municipaux en vue de promouvoir la sécurité des femmes. Le succès des efforts serait attribué selon les organisatrices, à la mobilisation centrale des groupes de femmes, des centres de femmes, des centres d'aide et d'écoute pour les femmes et CALACS.

proviennent de sources distinctes. Il devient done nécessaire pour les prévenir de les aborder séparément et différemment. L'influence des interventions en sécurité urbaine provient des disciplines de la criminologie, de la sociologie, de la psychologie, du féminisme, du droit, de l'économie et de l'urbanisme.

L'approche criminaliste étant la science qui aborde les fondements de la prévention de la criminalité, elle tente de retracer les liens de causes à effets du phénomène de la violence chez les individus qui l'utilisent et la perpétuent. Elle propose trois cibles d'intervention: 1) au niveau du développement de comportements délinquants chez les enfants (prévention développementale); 2) chez les individus qui ont un potentiel délinquant (prévention du potentiel délinquant); 3) chez les commetteurs de crimes, en rendant l'acte difficile à commettre (prévention situationnelle). Les agresseurs potentiels passent à l'acte lorsqu'il y a accessibilité (présence vulnérable) d'une cible et lorsque l'absence de têmoins fait diminuer le risque de se faire prendre par la police.

Les groupes de femmes, quant à eux, ont poussé plus loin la réflexion. Ils ont créé une approche nouvelle qui tient compte de la victime, de son sentiment d'aisance et de sécurité. Les aspects de l'environnement et les façons de dissuader les agresseurs de passer a l'acte tiennent alors compte du sentiment de sécurité de la femme, du respect et des droits de sa personne. Une femme devrait avoir le droit de circuler en toute sécurité le soir, elle devrait ne pas être obligée de dépendre de la présence d'un policier ou d'un accompagnateur. Cette vision a fait évoluer les mesures de sécurité urbaine selon une approche plus globale et holistique.

Les mesures correctives peuvent s inspirer des interventions des groupes de femmes et de l'approche criminaliste. Dans un premier temps, il s'agit d'amener les personnes concernées par la peur à identifier les éléments de l'environnement qui les insécurisent. Puis, déterminer avec elles et avec 1'apport d'experts, les causes de ces insécurites. Une fois les cau= ses identifiées, il faut mettre en place des mesures d'ordre individuel ou collectif qui visent la réduction des actes de violence ou des facteurs d'insécurité. Les résultats sont fonction des causes et du temps. Lorsque les causes sont liées à l'environnement, les mesures sont parfois simples et efficaces. Parfois, simplement le fait de parler en bien d'un lieu contribue positivement à sa réappropriation par le milieu.

\section{CONCLUSION}

L'insécurité est un sentiment qui paralyse. Les sources de l'insécurité sont multiples. Elles proviennent à la fois du vécu de la 
personne et, sur le plan collectif, des informations provenant des médias et de $l^{\top}$ organisation sociale des espaces urbains.

Le sentiment d'inséccuritế engendre plusieurs effets pervers. Il contribue à une baisse de la qualité de vie des personnes affectées, elles deviennent non seulement plus angoissées mais elles se déplacent moins et vivent l'isolement. L'insecurite contribue parfois au développement d'actes de violence urbaine en entrainant l'abandon de lieux publics, laissant toute la place aux manifestants d'actes de violence.

Le sentiment de sécurité a une valeur fondamentale sur la qualité de vie urbaine. Cette valeur peut se mesurer en terme de dollar si l'on calcule les effets de la présence des femmes et des personnes âgées fréquentant les lieux publics et voyageant dans une ville où il fait bon vivre. On peut evaluer le pouvoir d'attraction d'un quartier par les dépenses effectuées sur une rue commerciale par exemple ou par le développement résidentiel, etc.

La violence et l'insécurité provenant de sources distinctives constituent tout de même des problèmes très complexes. L'analyse de l'insécurité urbaine, de ses causes et de ses effets devraient guider le choix des intervenants.

Interventions. Un principe clé qu'il faudra garder en tête: comment rendre un site attrayant pour qu'il puisse y avoir l'achalandage d'une diversité de personnes où se dégage un sentiment de sécurité partagé. Les lieux publics doivent être parfois animés mais toujours invitants et propres. L'achalandage d'un lieu public le jour comme le soir est un indicateur de santé et de sécurité d'un secteur urbain capable d'offrir les éléments structurants d'une vitalité touristique.

\section{NOTES}

1 Nous verrons plus loin que l'insếcurité non seulement est une conséquence de la violence mais elle participe à l'émergence d"actes de violence urbaine sans en être la cause unique.

2 Carol Strike (1995), Les femmes agressees par des inconmus, Tendances sociales canadiennes, Statistique Canada, printemps.
3 Le milieu se caractérise par l'aménagement, les lieux publics, les personnes qui les fréquentent, les commerces et les diverses fonctions urbaines.

\section{BIBLIOGRAPHIE}

Catallo, Rose, Carolyn Whitzman (1994), Lessons from Success Stories: Making Communities Safer, Ville de Toronto, 80 pages.

Comité d"action femmes et sécurité urbaine (CAFSU) (1996), J'aime Montréal, bien sâr! Les prix de sécurité des femmes.

Cusson, M. et al. (1994), La planification et l'éva. luation de projets en prévention du crime. Ecole de criminologie de l"Université de Montréal, recherche commandée par le ministère de la Securité publique du Québec, 114 pages.

Développement québécois de la sécurité des femmes (1995), Esquisses de la sécurite des femmes dans les espaces publics: Québec, Rimouski, Chicoutimi, Sherbrooke, 81 pages.

Fedération canadienne des municipalités (1994), Conment rendre nos collectivités plus sâres pour les femmes: Guide a l'athention des dirigeants conmunautaires, $15 \mathrm{p}$.

Gouvernement du Québec (1993), Pour un Ouébec plus sécuritaire: partenaires en prévention, table ronde sur la prévention de la criminalité.

Kube, E., L. Schuster (1985), «Vandalism in a Urban Environment: Importance, Scop, and Context of the Phenomen: Possible Preventive Mensuress, Revue intemationale de criminologie et de police techinique, vol. 38, no 2, p. 139-161.

Maltais Munger, C. et al. (1995), Rapport de la Commission consultative. Fenmes et ville, ville de Québec, 208 pages.

McLeod, L. (1989), La femme dans la ville, conférence européenne et nord-américaine sur lạ sécurité et la prévention de la criminalité en milieu urbain du 11 au 14 octobre 1989,63 pages.

Secrétariat d'Ếtat du Canada et TANDEM Montréal (1992), J'accuse la peur, accès de la Conférence montréalaise sur les femmes et la sécurité urbaine, 30 mars, 58 pages.

TANDEM Montréal (1995), Le nom des hommes contre la violence, novembre.

The Metro Action Committee on Public Violence Against Women and Children (METRAC) (1992), Women's Safety Audir Guide, 47 pages.

The Safe City Committee (1988), Municipal Strategies for Preventing Public Violence Against Women, Toronto City Council, 16 pages.

The Safe City Committee (1988), Municipal Strategies for Preventing Public Volence Agains Women, Toronto City Council, September, 16 pages.
Wekerle, Gerda (1994), wiolence Against Women, Safe Cities: Canadian Federal and Municipal Initiatives s, conférence de l'OCDE, 4-6 octobre, women in the City: Housing, Services and the Urban Environment;, Paris.

\section{DES GUIDES PRATIQUES}

Guide d'aménagement sécuritaire des stationnements (1994), Comite femmes et ville, Senice de l'habitation et du developpement urbain, Ville de Montréal.

Par Sylvie Tremblay

Guide d'enquềte sur la sécurité des femmes en ville (1993). Service des loisirs, des parcs et du développement communautaire, Ville de Montréal, 44 pages.

Par A. Michaud, M.D. Lahaise et $M$. Henault

Take Back Totonto: A Guide to Preventing Violence Against Women in Your Community (1991). The City of Toronto, Safe City Committee and Healthy City Office. Par M. Gilmore

La trousse communautaire (1993), ministère des Approvisionnements et Services. Gouvernement du Canada, 173 p.

Par René Rouleau

\section{EN CONCLUSION}

Les fẻministes ont marqué des points importants en sécurité urbaine. Elles ont amené du sang neuf à la prévention situationnelle, qui s'était longtemps intéressée qu' à la protection de la population contre le vol. METRAC et les groupes de femmes qui ont suivi leur façon de faire et de penser, ont mis en lumière une série d'éléments environnementaux pouvant générer de l'insécurité. Elles ont également proposé des solutions pratiques et respectueuses de la liberté des femmes. Aujourd'hui, a Toronto comme à Montréal, plusieurs principes d'aménagement sont parfois pris en compte lors de l'élaboration de plans d'aménagements urbains et de transport public

Il est certain qu' une ville d'où se dégage un sentiment de sécurite attire le respect de l'environnement et des autres. Ceci contribue à une attitude de respect mutuel où le crime n'a pàs sa place et où les lieux publics sont' propriété collective des gens qui les fréquentent et les entretiennent. 